\title{
Cocaína: una revisión de la adicción y el tratamiento.
}

Cocaine: A review of addiction and treatment.

\author{
Irantzu González Llona a, Sumant Tumuluru b, Miguel Ángel González - Torres c, Moisés Gaviria d. \\ ${ }^{a}$ Servicio Vasco de Salud, Osakidetza, España ${ }^{b y}$ d Departamento de Psiquiatría. Universidad de Illinois \\ - Chicago, Estados Unidos. ${ }^{c}$ Servicio de Psiquiatría. Hospital Universitario de Basurto, España. Departa- \\ mento de Neurociencias. Universidad del País Vasco, España.
}

Correspondencia: Irantzu González.Llona (irantzu.gonzalezllona@osakidetza.net)

Recibido: 17/09/2014; aceptado: 03/04/2015

\begin{abstract}
RESUMEN: La cocaína es la droga responsable de más muertes en España. A pesar de una disminución del consumo en los últimos años, la prevalencia continúa siendo alta y es común hallarla en la práctica clínica. Si unimos a ello la peligrosidad del tóxico por su alta capacidad adictiva, las frecuentes y graves complicaciones que acarrea y la dificultad para su abandono, tenemos motivos de sobra para plantear una revisión tanto de su origen como de su estado actual, su abordaje clínico, y los retos para un futuro.

PALABRAS CLAVE: cocaína, revisión, origen, adicción, tratamiento.
\end{abstract}

ABSTRACT: Cocaine is the drug responsible for more deaths in Spain. Despite a decrease in consumption in recent years, the prevalence remains high and is common to find it in clinical practice. If you join it the danger of toxic because it's addictive high capacity, frequent and serious complications involved and the difficulty of abandonment, we have every reason to propose a revision of both its origin and its present, its clinical approach, and challenges for the future.

KEY WORDS: cocaine, review, origin, addiction, treatment.

\section{Introducción}

La cocaína es una droga ilegal altamente consumida con graves consecuencias orgánicas, psiquiátricas y sociales. Aunque cada vez la información disponible es mayor, es probable que el abordaje del problema aún diste de ser el adecuado dada la frecuencia del consumo y de sus complicaciones. El DSM IV-TR recoge de forma extensa diferentes trastornos relacionados con ella, bien por el propio consumo (intoxicación, abuso o dependencia), o inducidos por él (abstinencia, delírium, psicosis, ansiedad y alteración del ánimo, del sueño o sexual).

Es esencial conocer el contexto que envuelve al mundo de la droga y las consecuencias que acarrea para un mejor abordaje asistencial.

\section{Epidemiología}

En la Unión Europea la cocaína es, tras el cannabis, la droga ilegal de mayor consumo entre los adultos jóvenes. Alrededor de 7,5 millones de jóvenes europeos 
de entre 15 y 34 años la han probado en alguna ocasión. La prevalencia media de consumo alguna vez en la vida se sitúa en el 5,3\%. Sin embargo, cinco países superan esta cifra y, entre ellos, se encuentra España (1).

En España, entre 1994 y 2004 el uso de cocaína creció en todos los sectores de la población. En 2005, el 7\% de la población de 15 a 64 años la había probado alguna vez, el 3\% en los últimos doce meses y un $1,6 \%$ en el mes previo a ser encuestado. Tras una posterior estabilización (2005-2007), en el 2010, por primera vez el consumo descendió, encontrándose como excepción un incremento del consumo experimental entre los 20 y 45 años (de un 7\% en 2005 a un 10,2\% en 2010) (2).

Respecto al País Vasco, en 2010 y en el mismo grupo de edad, el 7,1\% había consumido alguna vez en su vida, el 1,6\% en el último año y el 1,1\% en el último mes (3).

Junto con la disminución general de su consumo encontramos que las solicitudes de tratamiento por problemas causados por el consumo de cocaína son las que registran mayor aumento respecto a otros tóxicos. En el año 2005, era ya responsable del $62,1 \%$ de las solicitudes de admisión a tratamiento por adicción por primera vez. Como contrapunto, la demanda de tratamiento suele ser tardía y se produce cuando ya ha generado conflictos personales, enfermedad psiquiátrica añadida, fracaso profesional, conflictos familiares o marginación social importante (1).

\section{Población consumidora}

El consumo de cocaína es más frecuente en hombres que en mujeres, y en la franja de edad de 15 a 34 años, con una edad media de inicio a los 20 años (1).

En lo que se refiere a los patrones de consumo, en nuestro entorno se ven básicamente dos: uno mayoritario caracterizado por un consumo de menor cantidad, generalmente por vía nasal, y otro minoritario que se da a menudo entre consumidores de heroína y que es un consumo más frecuente e importante que se lleva a cabo por vía intravenosa y pulmonar. Es frecuente el consumo asociado de otros tóxicos (1).

\section{Origen}

La cocaína es suministrada por Sudamérica, principalmente por Colombia. Se hizo popular en Estados Unidos y en las décadas de los 70 y 80 su consumo adquirió una extensión tal que fue considerada una epidemia. Las autoridades norteamericanas iniciaron una "guerra" contra la cocaína por la que su distribución hizo un giro 
ORIGINALES Y REVISIONES

hacia Europa en el año 2000. La existencia en España de una red de contrabando ya organizada previamente en torno al tabaco, de una amplia costa y de una baja vigilancia en algunos puertos permitió la rápida instauración de redes de tráfico.

De la cocaína que pasa por España buena parte es consumida en el país, lo que lo convierte en uno de los principales países consumidores de Europa y donde se está generando una cultura propia relacionada con el consumo muy en relación con la fiesta y la noche (4). Como dato guía, desde el año 1999 la cocaína es la droga ilegal más frecuentemente implicada en las urgencias hospitalarias relacionadas con el consumo agudo de drogas, habiendo llegando al 63,4\% en el año 2005 (1).

\section{Historia}

La cocaína o benzoilmetilecgonina es el principal alcaloide obtenido de las hojas del arbusto Erithroxylon Coca, originario de América del Sur y utilizado desde la antigüedad (5000 a. de C.) con fines mágico-religiosos, médicos y estimulantes por poblaciones indígenas. A través de una serie de procesos químicos que incluyen sustancias como queroseno y acido sulfúrico, la pasta de coca es extraída de las hojas y convertida en cocaína base (4).

En los países andinos ha servido para atenuar el cansancio y el mal de altura y anestesiar el aparato digestivo para no sentir hambre. Entre los Incas la coca era manjar, medicina, y moneda. En 1855, Albert Niemann, aisló el alcaloide principal de la coca: la cocaína, que se comercializó rápidamente como "alimento para los nervios" y como forma inofensiva de curar la tristeza, anestésico, tensor de las cuerdas vocales, y "para otorgar a las mujeres vitalidad y hermosura". (4)

Freud empezó a ensayar en 1880 su uso para el tratamiento de la neurastenia. Publicó "Über Coca", donde valoraba de forma positiva el uso de la cocaína para el tratamiento de la depresión, el nerviosismo, la adicción a la morfina, el alcoholismo, los trastornos digestivos, e incluso el asma. Finalmente admitió los efectos negativos de la cocaína tras experimentar con un colega suyo, que tras ser tratado por dolor con inyecciones subcutáneas terminó desarrollando paranoia, delirios y pérdida de autocontrol (5).

Aún así, su comercialización por diversos laboratorios farmacéuticos hizo que se volviera muy popular. En el mundo de la ficción, Sherlock Holmes se inyectaba cocaína cuando su mente se quedaba atascada en la resolución de algún caso, e incluso se dice que Robert L. Stevenson escribió su obra "El Dr. Jekyll y Mr. Hyde" bajo su influencia. En el último tercio del siglo XIX aparecieron bebidas con cocaína, la más conocida la Coca Cola, de la que se suprimió en 1909 sustituyéndose por cafeína (4).

Hacia 1900 pastillas y jarabes de cocaína se encontraban disponibles en farmacias y droguerías con libre acceso. La cocaína se hizo ilegal en Estados Unidos en 
el año 1914, y en España no fue hasta el Código Penal de 1944 cuando se consideró ilegal, especificándose los delitos sobre su venta ilícita, el tráfico, y el consumo (6).

\section{Efectos generales en el organismo}

La cocaína incrementa los niveles de Noradrenalina (NA), Dopamina (D) y en menor medida de Serotonina por bloqueo de su recaptación presináptica (7).

El aumento de Dopamina media la euforia que produce y parece el principal implicado en el mecanismo de adicción por la relación estrecha de este neurotransmisor con el sistema de recompensa cerebral. Con el consumo repetido se producen cambios en su disponibilidad: disminuye la síntesis de D y con ello sus niveles endógenos y su liberación, y se reduce la disponibilidad de los receptores D2/D3 (7).

El exceso de Noradrenalina es el responsable de la mayoría de los efectos físicos y de las complicaciones agudas de la cocaína: aumento de presión arterial, dilatación pupilar, sudoración, temblor, etc. El consumo crónico también produce una disminución de la biodisponibilidad de Serotonina. Por último, la disminución de la permeabilidad de la membrana a los iones $\mathrm{Na}+$ produce un bloqueo de la conducción nerviosa que explica su acción anestésica local.

Tanto las técnicas de neuroimagen como los estudios neuroendocrinos realizados avalan estos mecanismos (8-12):

Tabla 1.

Hallazgos con diferentes medios de respuestas orgánicas al consumo de cocaína. (8-12).

\begin{tabular}{|c|c|}
\hline Técnicas de neuroimagen & $\begin{array}{l}\text { Estudios neuroendocrinos: } \\
\text { Defectos funcionales }\end{array}$ \\
\hline $\begin{array}{l}\text { Incremento en los transportadores de D durante la } \\
\text { abstinencia aguda }\end{array}$ & Sistemas de D, 5-HT y NA directamente afectados. \\
\hline $\begin{array}{l}\text { Disminución de la afinidad del receptor D2 en adictos } \\
\text { desintoxicados }\end{array}$ & $\begin{array}{c}\text { Sistema de Glutamato, GABA y kappa Opioides } \\
\text { indirectamente afectados }\end{array}$ \\
\hline $\begin{array}{l}\text { Reducción del flujo sanguíneo cerebral en usuarios } \\
\text { crónicos }\end{array}$ & \\
\hline
\end{tabular}

Entre las drogas detectadas en las muertes directamente relacionadas con la reacción aguda del consumo de tóxicos ilegales en España, la cocaína es la más frecuente (1).

Los síntomas del consumo son consecuencia de su capacidad para estimular el SNC y el aparato cardiovascular y, como en otros estimulantes, las sobredosis pueden acabar produciendo efectos paradójicos de depresión neurológica y cardiovascular. 
ORIGINALES Y REVISIONES

Respecto al Sistema Cardiovascular lo más frecuente es que el paciente consulte por palpitaciones. Suele objetivarse taquicardia sinusal y puede añadirse hipertensión arterial. A veces aparece dolor torácico de características anginosas. En el ECG pueden observarse arritmias y trastornos de isquemia-lesión, que pueden llegar al infarto agudo de miocardio. Menos frecuentes son la hipotensión arterial y el shock (signos de extrema gravedad), el aneurisma aórtico agudo, las bradicardias con síndromes de QT largo y disección aórtica. Incrementa el riesgo de IAM y de muerte repentina siendo el fallo cardíaco la causa de más frecuente muerte asociada al consumo (13).

Sobre el SNC, y aunque la mayoría de los pacientes sean remitidos por inquietud o agitación (13), causa midriasis, mioclonias, convulsiones y coma de forma aguda, y deterioro cognitivo leve pero duradero y limitación de la plasticidad neuronal de forma crónica. También aumenta el riesgo de traumatismo craneal y accidentes cerebrovasculares hemorrágicos y/o isquémicos.

Además, los usuarios de cocaína intravenosa tienen mayor probabilidad de contraer VIH y Hepatitis $\mathrm{C}$ y de presentar infiltrados pulmonares, edema pulmonar, y exacerbación asmática severa (14).

Otros síntomas pueden ser sudoración fría, náuseas, vómitos, sequedad bucal, isquemia intestinal, dolores musculares, rabdomiolisis, insuficiencia renal secundaria, alteraciones del equilibrio ácido-base y trastornos funcionales hepáticos y pancreáticos. En algunos pacientes que se inyectan cocaína, se ha constatado el desarrollo de un cuadro fulminante de shock y coagulación intravascular diseminada. También es posible el desarrollo de una hipertermia maligna-like (13).

\section{Efectos psiquiátricos en el organismo}

Respecto a sus efectos psiquiátricos, es causa y precipitante de cuadros de ansiedad, compulsiones, ideación de tipo paranoide, cuadros psicóticos, desarrollo de ideas delirantes... tanto en su uso agudo como crónico (14).

El consumo de cocaína se asocia frecuentemente a comorbilidad con enfermedades mentales y consumo de otros tóxicos. En un estudio realizado a 303 usuarios de crack (cocaína fumada), se vieron los siguientes resultados (15): 
Gráfica 1.

Comorbilidad psiquiátrica en usuarios de cocaína. (15)

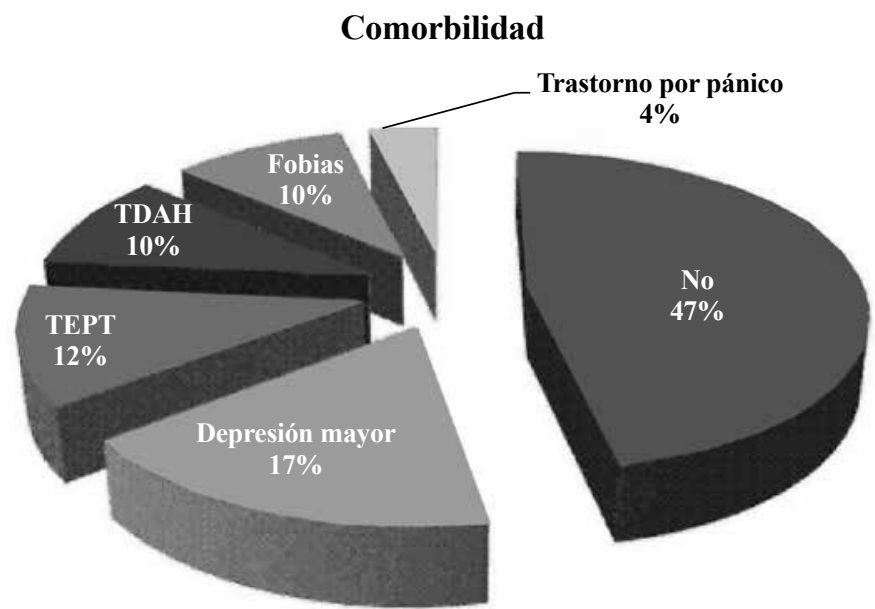

Además, el 45,3\% de los consumidores eran también dependientes de opiáceos y el $30,1 \%$ de marihuana.

Cocaina y Psicosis

La psicosis paranoide inducida por cocaína (síntoma más característico) es un aparatoso cuadro psiquiátrico que presenta hasta el $70 \%$ de los intoxicados y entre el $53 \%$ y el $68 \%$ de los adictos. Su aparición suele variar entre 25 y 57 meses desde el inicio del consumo regular, con una media en torno a los tres años (16). Hay mayor predisposición en hombres que en mujeres, en personas que previamente han presentado cuadros similares (17) y en consumidores vía iv respecto a vía nasal (18).

Habitualmente cursa con agresividad y agitación, delirios paranoides y celotípicos y alteraciones en la esfera afectiva. Las alucinaciones no son extrañas (son clásicas aunque raras las visuales de infestación parasitaria) y con frecuencia se ven estereotipias motoras.

En la urgencia psiquiátrica, ante un cuadro psicótico en un paciente con antecedentes de abuso de sustancias psicoactivas hay que pensar siempre en el origen tóxico hasta que se demuestre lo contrario. Así se reduce el posible daño resultante de no tratar una intoxicación o un síndrome de abstinencia, o el derivado de una exposición innecesaria de los pacientes al tratamiento neuroléptico (19).

Cocaina y esquizofrenia

El abuso de cocaína disminuye la efectividad de los neurolépticos (20) y multiplica por cuatro el riesgo de aparición de distonía aguda y discinesia tardía (21). Además, se ha relacionado con el incumplimiento del tratamiento en estos pacientes, lo que junto con una frecuencia de consumo entre los mismos situada entre el 22 y $31 \%$ (22), supone mayor número de reagudizaciones y mayor gravedad de la sintomatología positiva y negativa (23). 
ORIGINALES Y REVISIONES

\section{Cocaina y Patología Afectiva}

En pacientes con patología afectiva la prevalencia de consumo de cocaína varía entre el 33-50\% siendo más frecuente en mujeres. Concretamente en el Trastorno Bipolar, se ha relacionado con un inicio más precoz y una peor evolución del mismo, con mayor número de episodios mixtos y episodios maníacos más graves (24). Además, el consumo empeora toda sintomatología depresiva (25).

Estos pacientes presentan mejor respuesta a eutimizantes anticonvulsivantes que a sales de litio (26), pudiendo aplicarse además los datos referidos anteriormente sobre efectos adversos de neurolépticos en esquizofrenia (20-21).

\section{Diagnóstico}

Para diagnosticar trastornos por consumo de cocaína es necesario realizar una historia clínica completa del consumo de cocaína y otros tóxicos, incluyendo circunstancias de consumo, efectos, vías, frecuencia, cantidad y consecuencias (biológicas y psicosociales) (27).

Así mismo, en el caso de que existan antecedentes o clínica psiquiátrica, debemos prestar una atención particular a la secuencia temporal entre los mismos y el consumo. Así, se podrá evitar el error de tratar con psicofármacos síntomas limitados secundarios al propio consumo (27).

Es básico además realizar un examen físico completo y exámenes complementarios para descartar infección por VIH y otras ETS, alteraciones cardíacas, déficits vitamínicos y desnutrición (27).

A la hora de clasificar la patología relacionada con el consumo de cocaína, el DSM IV-TR (28) recoge dos apartados: 
Tabla 2.

Apartados diagnósticos cocaína DSM IV-TR (28)

1. Trastornos por consumo de cocaína

F14.2x Dependencia de cocaína [304.20]

F14.1 Abuso de cocaína [305.60]

2. Trastornos inducidos por cocaína

F14.00 Intoxicación por cocaína [292.89]

F14.04 Intoxicación por cocaína, con alteraciones perceptivas

F14.3 Abstinencia de cocaína [292.0]

F14.03 Delirium por intoxicación por cocaína [292.81]

F14.51 Trastorno psicótico inducido por cocaína, con ideas delirantes [292.11] Especificar si: De inicio durante la intoxicación

F14.52 Trastorno psicótico inducido por cocaína, con alucinaciones [292.12] Especificar si: De inicio durante la intoxicación

F14.8 Trastorno del estado de ánimo inducido por cocaína [292.84] Especificar si: De inicio durante la intoxicación/De inicio durante la abstinencia

F14.8 Trastorno de ansiedad inducido por cocaína [292.89] Especificar si: De inicio durante la intoxicación de inicio/De inicio durante la abstinencia

F14.8 Trastorno sexual inducido por cocaína [292.89] Especificar si: De inicio durante la intoxicación

F14.8 Trastorno del sueño inducido por cocaína [292.89] Especificar si: De inicio durante la intoxicación/De inicio durante la abstinencia

F14.9 Trastorno relacionado con cocaína no especificado [292.9]

\section{Dependencia a cocaína}

Según el DSM IV-TR, se define la dependencia como un patrón desadaptativo de consumo que conlleva un deterioro o malestar clínicamente significativos, expresado por tres o más de los siguientes ítems en algún momento de un período continuado de 12 meses:

1. Tolerancia, definida por cualquiera de los siguientes ítems:

- Una necesidad de cantidades marcadamente crecientes de la sustancia para conseguir la intoxicación o el efecto deseado.

- El efecto de las mismas cantidades de sustancia disminuye claramente con su consumo continuado. 
ORIGINALES Y REVISIONES

2. Abstinencia, definida por cualquiera de los siguientes ítems:

- Síndrome de abstinencia característico.

- Se toma la misma sustancia (o una muy parecida) para aliviar o evitar los síntomas de abstinencia.

- La sustancia es tomada con frecuencia en cantidades mayores o durante un período más largo de lo que inicialmente se pretendía.

- Existe un deseo persistente o esfuerzos infructuosos de controlar o interrumpir el consumo de la sustancia.

- Se emplea mucho tiempo en actividades relacionadas con la obtención de la sustancia, en el consumo de la sustancia o en la recuperación de los efectos de la sustancia. - Reducción de importantes actividades sociales, laborales o recreativas debido al consumo de la sustancia.

- Se continúa tomando la sustancia a pesar de tener conciencia de problemas psicológicos o físicos recidivantes o persistentes, que parecen causados o exacerbados por el consumo de la sustancia.

Se realizarán especificaciones sobre el estado actual del consumo y sobre si existe dependencia física.

Abuso de cocaina

En el mismo manual (28), se define como un patrón desadaptativo de consumo, cuyos síntomas no han cumplido nunca los criterios para la dependencia mencionados anteriormente, y que conlleva un deterioro o malestar clínicamente significativos, expresado por uno o más de los ítems siguientes durante un período de 12 meses: 1. Consumo recurrente de sustancias, que da lugar al incumplimiento de obligaciones en el trabajo, la escuela o en casa.

2. Consumo recurrente de la sustancia en situaciones en las que hacerlo es físicamente peligroso.

3. Problemas legales repetidos relacionados con la sustancia.

4. Consumo continuado de la sustancia, a pesar de tener problemas sociales continuos o recurrentes o problemas interpersonales causados o exacerbados por los efectos de la sustancia.

\section{Intoxicación por cocaína}

La respuesta a la cocaína depende de la dosis, vía de administración, experiencias previas del sujeto, el ambiente, y no podemos olvidar que existe una parte de respuesta única de cada consumidor.

Con cualquier dosis pueden verse euforia que puede derivar en disforia, aumento de la comunicación verbal, autoconfianza y actividad sexual, inquietud psicomotriz, anorexia, insomnio, euforia, pupilas midriáticas, taquicardia, hipertensión y crisis con agitación. Con dosis altas se hacen más frecuentes la diaforesis, temblores, confusión y/o hiperactividad, aumentando de forma muy llamativa el riesgo de convulsiones, hemorragia cerebral, arritmias, muerte súbita... 
Con el tiempo, aparece tolerancia a los efectos euforizante y anorexígeno (29). Se calcula que hasta una de cada tres visitas al servicio de urgencias relacionadas con drogas está motivada por un consumo de cocaína. Los motivos de consulta más frecuentes presentados en pacientes atendidos en los servicios de urgencias por intoxicación o sobredosis de esta droga son los siguientes (30):

Tabla 3

Motivos de consulta más frecuentes en pacientes atendidos en Servicio de Urgencias por Intoxicación por Cocaína. (30).

\begin{tabular}{|c|c|}
\hline Psiquiátricos & $83(54,6 \%)$ \\
\hline Ansiedad & $40(26,3 \%)$ \\
\hline Agitación & $33(21,7 \%)$ \\
\hline Delirio & $6(3,9 \%)$ \\
\hline Alucinaciones & $4(2 \%)$ \\
\hline Cardiovasculares & $38(25 \%)$ \\
\hline Palpitaciones & $21(13 \%)$ \\
\hline Dolor torácico & $17(11,1 \%)$ \\
\hline Neurológicos & $19(12,5 \%)$ \\
\hline Disminución del nivel de conciencia & $11(7 \%)$ \\
\hline Movimientos anormales & $5(3 \%)$ \\
\hline Convulsiones & $2(1,9 \%)$ \\
\hline Preocupación por consumo & $20(13,1 \%)$ \\
\hline Tentativa de suicidio & $11(7,2 \%)$ \\
\hline
\end{tabular}

Hoy en día, por suerte, los test bioquímicos pueden ser utilizados para medir la presencia y la concentración de metabolitos de la cocaína en orina (más habitual), saliva, sangre o pelo (31), lo que contribuye a un mejor diagnóstico diferencial de la etiología del cuadro.

\section{Abstinencia a cocaína}

$1^{a}$ fase: El crash sigue inmediatamente a la discontinuación del consumo, teniendo una duración de 9 horas a 5 días. Los síntomas físicos son predominantes, mientras que el deseo de consumo va disminuyendo progresivamente.

$2^{a}$ Fase: abstinencia propiamente dicha. Dura de 1 a 10 semanas.

-Temprana. Normalización del ritmo de sueño y del estado de ánimo, baja ansiedad y bajo deseo de cocaína.

-Media y tardía. Disforia, anhedonia, anergia, incremento de ansiedad, irritabilidad, intenso deseo de cocaína (craving) condicionado por sucesos que lo exacerban.

$3^{a}$ Fase: extinción. Tiene una duración indefinida, pudiendo considerarse que se mantiene toda la vida. La caracterizan eutimia, recuerdo de los efectos agradables de la cocaína y deseo ocasional de cocaína en relación con estímulos condicionados. 
ORIGINALES Y REVISIONES

A menudo no hay síntomas visibles de la abstinencia como vómitos o temblor, que pueden acompañar a la abstinencia de drogas como la heroína, y sin embargo el nivel de craving, irritabilidad y síndrome depresivo diferido rivaliza o excede a los de la abstinencia a otras drogas (32).

\section{Cocaina y DSM-5}

Lejos de pretender revisar los cambios detallados entre el manual diagnóstico anterior y éste, como principales diferencias cabrían destacar las siguientes:

La distinción entre Abuso y Dependencia desaparece, apareciendo el diagnóstico único de Trastorno por consumo de estimulantes (con subapartado de cocaína). Permanecerían la Intoxicación por estimulantes y Abstinencia de estimulantes.

Los trastornos mentales inducidos por cocaína podrían ser diagnosticados pero se incluirían en los apartados correspondientes a la psicopatología y no el consumo (por ejemplo, Trastorno psicótico inducido por sustancias, dentro del capítulo Espectro de la esquizofrenia y otros trastornos psicóticos).

\section{Tratamiento farmacológico de la intoxicación aguda}

Es básico tomar siempre las constantes vitales del paciente y monitorizar el ECG. La sobredosis de cocaína carece de antídoto y por ello requiere un tratamiento sintomático:

En caso de agitación, es necesario descartar hipoglucemia, hipoxia y asociación con otros psicoestimulantes. Se podrá administrar diazepam, midazolam o clonazepam iv lento y se deberá evitar el riesgo de autolesiones con barandillas, contención física si precisa, etc.

Tanto si se objetivan convulsiones, como en caso de taquicardia, hipertensión o arritmias, el tratamiento de elección es el diazepam (sublingual, oral o iv lento). $\mathrm{Si}$ coexisten hipertensión y arritmias, se utilizará labetalol, estando el propranolol contraindicado ya que puede desencadenar o empeorar la hipertensión arterial.

Por otra parte, la bradicardia con QT largo requiere taquicardizar al paciente con una perfusión de isoprenalina y descartar una hipomagnesemia. Para la taquicardia ventricular se ha propuesto el uso de fenitoína (33). 


\section{Tratamiento farmacológico de la dependencia}

Históricamente los intentos de encontrar una medicación para tratar la dependencia a cocaína se han focalizado en el bloqueo de los receptores postsinápticos de D y la afinidad a los autorreceptores presinápticos que regulan su liberación. Hallazgos recientes se han centrado en otros sistemas de NT que han ofrecido avances significativos (34), y de hecho las medicaciones más prometedoras modifican más la neurotransmisión de NA que de D (35).

Han sido probadas muchas medicaciones sin haber sido hallada aún una realmente eficaz (36).

Tabla 4.

Estudios farmacológicos más relevantes en la dependencia a cocaína (36).

\begin{tabular}{|c|c|c|c|c|}
\hline Familia & Fármaco & Objetivo primario & Dependencia a droga & Eficacia $(+/-)$ \\
\hline \multirow{6}{*}{ Antidepresivo } & Desipramina & $\mathrm{NE}$ & Cocaína & - \\
\hline & Fluoxetina & $5 \mathrm{HT}$ & Cocaína & - \\
\hline & Bupropion & NE, D & Cocaína, Metanfetaminas & - \\
\hline & Venlafaxina & NE, 5HT, D & Cocaína & - \\
\hline & Sertralina & $5 \mathrm{HT}$ & Cocaína & - \\
\hline & Imipramina & NE, $5 \mathrm{HT}$ & Cocaína & - \\
\hline \multirow{15}{*}{$\begin{array}{c}\text { Estabilizadores del } \\
\text { ánimo / Anticonvul- } \\
\text { sivantes }\end{array}$} & Carbamazepina & Sodio & Cocaína & - \\
\hline & Litio & Inositol P3 & Cocaína & - \\
\hline & Fenitoína & Sodio, Calcio & Cocaína & - \\
\hline & Vigabatrina & GABA & Cocaína & + \\
\hline & Tiagabina & GABA & Cocaína & “+/_“" \\
\hline & Baclofeno & GABA & Cocaína & "+/_“ \\
\hline & Bromocriptina & $\mathrm{D}$ & Cocaína, Metanfetaminas & “+/_“ \\
\hline & Pergolida & $\mathrm{D}$ & Cocaína & - \\
\hline & Amantadina & $\mathrm{D}$ & Cocaína & - \\
\hline & Aripiprazol & $\mathrm{D}$ & Cocaína, Metanfetaminas & - \\
\hline & Metilfenidato & $\mathrm{D}$ & Cocaína, Anfetaminas & + \\
\hline & Levodopa / Carbodopa & $\mathrm{D}$ & Cocaína & + \\
\hline & Modafinilo & $\mathrm{D}$ & Cocaína, Metanfetaminas & + \\
\hline & Disulfiram & $\mathrm{D}, \mathrm{NE}$ & Cocaína & - \\
\hline & Nimodipina & Calcio & Cocaína & - \\
\hline
\end{tabular}


ORIGINALES Y REVISIONES

\section{Tratamiento no farmacológico de la dependencia}

Tabla 5 .

Tratamientos no farmacológicos más relevantes en la dependencia a cocaína (37).

\begin{tabular}{|c|c|c|}
\hline \multicolumn{3}{|c|}{ Tratamientos psicológicos de la dependencia de cocaína } \\
\hline $\begin{array}{l}\text { Tratamientos } \\
\text { eficaces }\end{array}$ & $\begin{array}{l}\text { Tratamiento manualizado y protocolizado con } \\
\text { psicólogos entrenados y un número suficiente } \\
\text { de evidencias científicas derivadas de varios en- } \\
\text { sayos clínicos prospectivos controlados, rando- } \\
\text { mizados y realizados con un número suficiente } \\
\text { de pacientes o bien metanálisis, que demuestren } \\
\text { su eficacia en la prevención de recaídas }\end{array}$ & $\begin{array}{l}\text { Enfoque de Refuerzo Comunitario + } \\
\text { "vouchers" }\end{array}$ \\
\hline $\begin{array}{l}\text { Tratamientos proba- } \\
\text { blemente Eficaces }\end{array}$ & $\begin{array}{l}\text { Tratamientos con evidencias que sustentan su } \\
\text { utilización en dependientes de cocaína, aunque } \\
\text { se precisa de un mayor número de ensayos clí- } \\
\text { nicos controlados o metanálisis que confirmen } \\
\text { los resultados iniciales }\end{array}$ & $\begin{array}{l}\text { - Counseling, asesoramiento o } \\
\text { consejo individual y grupal. } \\
\text { - Exposición sistemática a estímulos } \\
\text { y entrenamiento en habilidades de } \\
\text { afrontamiento. } \\
\text { - Terapia Cognitivo-conductual } \\
\text { (TCC). } \\
\text { - Prevención de recaídas (P/R). } \\
\text { - Entrevista motivacional (EM). }\end{array}$ \\
\hline $\begin{array}{l}\text { Tratamientos que } \\
\text { requieren más inves- } \\
\text { tigación }\end{array}$ & $\begin{array}{l}\text { Tratamientos que requieren mayor protocoliza- } \\
\text { ción y un número de ensayos clínicos cerrados, } \\
\text { con un número suficiente de pacientes, para } \\
\text { confirmar la evidencia de su eficacia. Trata- } \\
\text { mientos indicados para problemas comórbidos } \\
\text { específicos }\end{array}$ & $\begin{array}{l}\text { - Terapia cognitivo-conductual para } \\
\text { el desorden por estrés post-traumático } \\
\text { (PTSD). } \\
\text { - Terapia familiar estratégica breve } \\
\text { (BSFT). } \\
\text { - Terapia de apoyo expresiva (TSE). } \\
\text { - Terapia interpersonal (TIP). }\end{array}$ \\
\hline Tratamientos útiles & $\begin{array}{l}\text { Tratamientos psicológicos clásicos del ámbito } \\
\text { de la salud mental que también se utilizan en } \\
\text { dependientes de cocaína en los dispositivos } \\
\text { de tratamiento y contribuyen a la estabilidad } \\
\text { a largo plazo del paciente aunque carecen de } \\
\text { ensayos clínicos rigurosos y por tanto de evi- } \\
\text { dencias que avalen su utilización }\end{array}$ & $\begin{array}{l}\text { - Psicoanálisis y terapias dinámicas. } \\
\text { - Terapia de pareja. } \\
\text { - Terapia familiar. } \\
\text { - Terapias grupales no estructuradas. }\end{array}$ \\
\hline
\end{tabular}

Múltiples intervenciones psicoterapéuticas han sido evaluadas. Cabe mencionar que todas ellas pueden realizarse en formato individual o grupal. Procedemos a resumir las más estudiadas.

1. Enfoque de Refuerzo Comunitario o Comunidad Terapéutica: con un grado de recomendación A, está indicado para pacientes con dependencia grave, pobre control de impulsos, con historia de fracasos en tratamientos menos intensivos y con escaso apoyo social.

2. Tratamiento cognitivo - conductual (TCC): Grado de recomendación A. Es eficaz en el tratamiento de las conductas adictivas). Las bases son el análisis funcional de la conducta adictiva, el entrenamiento práctico de habilidades para el recono- 
cimiento y afrontamiento del "craving", la solución de problemas, la identificación y anticipación de situaciones de riesgo, el reconocimiento de decisiones aparentemente irrelevantes y habilidades para rechazar el consumo, el control de los procesos cognitivos relacionados con el consumo de drogas, el control y programación de las actividades.

3. Prevención de Recaídas, como variante más específica del TCC: Grado de Recomendación B. En general, el tratamiento tiene dos componentes esenciales: el uso del análisis funcional del consumo y el entrenamiento en habilidades. El objetivo es que el paciente aprenda a anticipar y enfrentarse a las presiones y problemas que pueden conducirle a una recaída.

4. Entrevista motivacional: se le atribuye un grado de recomendación B. Es eficaz en primera fase y acompañando a otras intervenciones. Está indicada en pacientes con abuso (NO dependientes) y aumenta la adherencia y cumplimiento del tratamiento. Según el nivel de evidencia 1 está más indicada cuanto menor sea la motivación para el cambio en la línea base, y es apta para favorecer el inicio de un tratamiento, para los programas de reducción del daño y para favorecer la adherencia a otras modalidades psicoterapéuticas.

5. Terapia interpersonal: Grado de recomendación C ya que mejora los resultados a largo plazo. Se basa en la idea de que el problema de la droga surge y se mantiene en un contexto de relaciones interpersonales.

6. Intervención familiar: Grado de recomendación C. Describe las conductas en términos de relaciones interpersonales al mismo tiempo que estudia las posibles modificaciones o cambios que movilicen las posiciones o interacciones que dificultan un desarrollo armónico y coherente.

7. Terapia psicoanalítica: Grado recomendación C. Indicada una vez que la persona haya conseguido la abstinencia, en "estados avanzados de recuperación, para tratar rasgos de personalidad anómalos, asociados o previos a la adicción".

\section{Enfoque hacia el futuro}

Las adicciones son cada vez más ampliamente reconocidas como una enfermedad crónica en la que las recaídas son comunes, con lo que el foco se centra cada vez más en la prevención y reducción de daños.

El rumbo de las investigaciones hace pensar que el hallazgo de un tratamiento eficaz se acerca. Las medicaciones con acciones neurotróficas podrían revertir los cambios inducidos por drogas, estimular el funcionamiento cognitivo y reducir el estrés oxidativo, y cabe pensar que con el tiempo se conseguirán objetivos farmacoterapeúticos como bloquear o revertir la plasticidad sináptica asociada a la dependencia a drogas. 
ORIGINALES Y REVISIONES

Para ello, aún se necesita tomar un abordaje más individualizado basado en el subtipo de dependencia a droga, género, raza, comorbilidades médicas y psiquiátricas, deficiencias funcionales cerebrales, perfil farmacogenético...

\section{BIBLIOGRAFÍA}

(1) Actas de reuniones. Ministerio de Sanidad y Consumo, editor. Informes de la Comisión Clínica de la Delegación del Gobierno para el Plan Nacional sobre Drogas: Cocaína; 2008; España.

(2) Informe de encuesta. Ministerio de Sanidad y consumo, editor. Encuesta Domiciliaria sobre Alcohol y Drogas en España (EDADES); 2011; España.

(3) Informe de estudio. Centro de Documentación y Estudios, SiiS. Secretaria General de Drogodependencias del Gobierno Vasco, editor. Informe Euskadi y Drogas; 2010; España.

(4) Pascual Pastor F. Aproximación histórica a la cocaína: De la coca a la cocaína. Adicciones 2001;13(supl 2): 7-22.

(5) Freud, Sigmund. Escritos sobre la cocaína. Editorial Anagrama, 1980.

(6) Herrero Álvarez S. Cocaína en el derecho penal español. Adicciones 2001;13(supl 2): 227-247.

(7) Haile CN, Mahoney JJ, Newton TF, De La Garza R. Pharmacotherapeutics directed at deficiencies associated with cocaine dependence: focus on dopamine, norepinephrine and glutamate. Pharmacol Ther. 2012;134(2):260-77

(8) Malison RT, Best SE, Van Dyck CH, McCance EF, Wallace EA, Laruelle M, Baldwin RM, et al. Elevated striatal dopamine transporters during acute cocaine abstinence as measured by 123I beta-CIT SPECT. Am J Psychiatry. 1998;155(6):832-4.

(9) Volkow ND, Wang GJ, Fowler JS, Gatley SJ, Ding YS, Logan J, et al. Relationship between psychostimulant-induced high and dopamine transporter occupancy. J.Proc Natl Acad Sci U S A. 1996 Sep 17;93(19):10388-92.

(10) Holman BL, Carvalho PA, Mendelson J, Teoh SK, Nardin R, Hallgring E, Hebben $\mathrm{N}$, Johnson KA. Brain perfusion is abnormal in cocaine-dependent polydrug users: a study using technetium-99m-HMPAO and ASPECT. J Nucl Med. 1991 Jun;32(6):1206-10.

(11) Aronson SC, Black JE, McDougle CJ, Scanley BE, Jatlow P, Kosten TR,Heninger GR, et al. Serotonergic mechanisms of cocaine effects in humans. Psychopharmacology (Berl). 1995 May;119(2):179-85.

(12) Fischman MW, Schuster CR, Javaid J, Hatano Y, Davis J. (1985) Acute tolerance development to the cardiovascular and subjective effects of cocaine. J Pharmacol Exp Ther. 1985 Dec;235(3):677-82.

(13) Fiala M, Eshleman AJ, Cashman J, Lin J, Lossinsky AS, Suarez V, et al. Cocaine increases human immunodeficiency virus type 1 neuroinvasion through remodeling brain microvascular endothelial cells. J Neurovirol. 2005 Jul;11(3):281-91.

(14) Roncero J, Ramos J.A, Collazos F, Casas M. Complicaciones psicóticas del consumo de cocaína. Adicciones 2001;13(supl 2): 179-190.

(15) Falck RS, Wang J, Siegal HA, Carlson RG. The prevalence of psychiatric disorder among a community sample of crack cocaine users: an exploratory study with practical implications. J Nerv Ment Dis. 2004 Jul;192(7):503-7. 
(16) Satel SL, Edell WS. Cocaine induced paranoia and psychosis proneness. Am J Psychiatry 1991;148:1708-1711.

(17) Post RM. Weiss SRB, Pert A, Uhde RW. Chronic Cocaine administration: Sensitization and kindling effects. En Fisher S, Raskin A, Uhlenhuth EH, editores. Cocaine: Clinical and Biobehavioral Aspects. New York: Oxford University Press, 1987; p. 109-173.

(18) Brady KT, Lydiard RB, Malcolm R, Ballenger JC. Cocaine-Induced Psychosis. J Clin Psychiatry; 1991;52: 509-512.

(19) Rosenthal RN, Miner CR. Differential Diagnosis of substance-Induced Psychosis and Schizophrenia in patients with substance use disorders. Schizophr Bull. 1997;23(2):187-93.

(20) Laudet A.B. Magura S., Vogel H.S., Knight E. Recovery challenges among dually diagnosed individuals. J Subst Abuse Treat. 2000 Jun;18(4):321-9.

(21) Van Harten PN, van Trier JC, Horwitz EH, Martos GE, Hoek HW. (1998) Cocaine as a risk factor for neuroleptic-induced acute dystonia. J Clin Psychiatry Mart;59(3):128-30.

(22) Batel P. Addiction and schizophrenia. Eur Psychiatry. 2000;15:115-22.

(23) North CS, Pollio DE, Smith EM, Spitznagel EL. Correlates of early onset and chronicity of homelessness in large urban homeless population. J Nerv Ment Dis. 1998 Jul;186(7):393-400.

(24) Strakowsky SS, Tohen M, Stoll Al, Faedda GL, Goodwin DC. Comorbidity in mania at first hospitalization. Am J Psychiatry. 1992;149:554-6.

(25) Weddington WW, Brown BS, Haertzen CA. Changes in mood, craving, and sleep during short-term abstinence reported by male cocaine addicts. A controlled, residential study. Arch Gen Psychiatry. 1990;47:861-8.

(26) Goldberg JF, Garno JL, Leon AC, Kocsis JH, Portera L. A history of substance abuse complicates remission from acute mania in bipolar disorder. J Clin Psychiatry. 1999 Nov; 60(11):73340 .

(27) Caballero Martinez, L. Adicción a Cocaína: Neurobiología clínica, diagnóstico y tratamiento. Ministerio de Sanidad y Consumo, editor; 2005; España.

(28) DSM-IV. Manual diagnóstico y estadístico de los trastornos mentales. Ed. Masson; 1995; España.

(29) Schuster CR. Monitoring the impact of cocaine. JAMA. 1991;266(16):2273.

(30) Sanjurjoa E, Montoria E, Noguéb S, Miquel Sánchez M, Munnéb P. Urgencias por cocaína: un problema emergente. Med Clin (Barc). 2006;126(16):616-9.

(31) Yoon JH, Newton TF, Haile CN, Bordnick PS, Fintzy RE, Culbertson C, et al. Effects of D-cycloserine on cue-induced craving and cigarette smoking among concurrent cocaine- and nicotine-dependent volunteers. Addict Behav. 2013 Feb;38(2):1518-26.

(32) Llopis Llácer JJ. Dependencia, intoxicación aguda y síndrome de abstinencia por cocaína. Adicciones 2001;13(supl 2): 147-166.

(33) Protocolos de Tratamiento del Área de Vigilancia Intensiva. Unidad de Toxicología Clínica, Servicio de Urgencias. Hospital Clinic Barcelona; 2000. Disponible en http://www. scfarmclin.org/toxicologia.php?idioma=cat.

(34) Rothman RB, Baumann MH, Dersch CM, Romero DV, Rice KC, Carroll FI, et al. Amphetamine-type central nervous system stimulants release norepinephrine more potently than they release dopamine and serotonin. Synapse. $2001 \mathrm{Jan} ; 39(1): 32-41$.

(35) Weinshenker D, Schroeder JP. There and back again: a tale of norepinephrine and drug addiction. Neuropsychopharmacology. 2007;32(7):1433-51. 
ORIGINALES Y REVISIONES

(36) Newton TF, De La Garza R, Brown G, Kosten TR, Mahoney JJ, Haile CN. Noradrenergic $\alpha$ receptor antagonist treatment attenuates positive subjective effects of cocaine in humans: a randomized trial. PLoS One. 2012;7(2):e30854.

(37) Guía-Protocolo de Actuación en Personas con Trastorno por Consumo de Cocaína. Servicio Extremeño de Salud; 2011. Disponible en: http://padib.caib.es/sacmicrofront/archivopub.do ?ctrl=MCRST352ZI108651\&id=108651. 